\title{
Do We See Magnetic Effects in Dwarf Nova Outbursts?
}

\author{
N. Vogt ${ }^{1,2}$, E. Meyer-Hofmeister ${ }^{1}, F$. Meyer $^{1}$ \\ 1 Max-Planck-Institut für Astrophysik, 85740 Garching, Germany \\ ${ }^{2}$ Universidad Catolica de Chile, Santiago, Chile
}

Many observations indicate that fast rotating late type stars show magnetic activity. We therefore argue that some of the secondary stars in cataclysmic binaries might also have magnetic fields. Such magnetic fields would reach over the accretion disk around the white dwarf primary. We investigate their effect on dwarf nova outbursts. The magnetic field lines will penetrate the disk and remove angular momentum. This shifts the accumulation of mass towards the inner disk, closer to the white dwarf, and therefore leads to a different outburst behaviour, which can be recognized in observed light curves of dwarf novae. If a magnetic field of the order 50 - 100 gauss is acting on the accretion disk, we expect narrower and more frequent outbursts as compared to the non-magnetic case. Outburst records of three dwarf novae above the period gap $(P>3 h)$, whose long-term light curves are well covered, were used to search for traces of magnetic activity. All three cases display a pronounced bimodality in the distribution of their outburst width, i.e. either narrow or wide outbursts occur. We found evidence for a cyclic behaviour in one case, SS Aur: possible "magnetic episodes" repeating every 18 years reveal epochs with abnormally frequent narrow outbursts and nearly or totally missing wide ones. There are also indications for a similar behaviour of SS Cyg with a 7-years cycle, but with less pronounced periodicity. The third case, U Gem, does not show clear evidence of magnetic activity although we found some indications for a transitory oscillation of the width of wide outbursts after 1926, with a period of 13.6 years. The behaviour of SS Aur and SS Cyg resembles the theoretical predictions, there is, however, an important difference: Throughout the entire cycle, marked by the "magnetic episodes", neither wide nor narrow outbursts alter their mean light curves: magnetic fields seem to affect only the observed proportion in the frequency of both outburst types without altering the light curves of individual outbursts. Remarkably, most of the "anomalous outbursts" (which are characterized by an abnormally slow rising branch to an outburst) occur also at or near the "magnetic episodes".

The complete version of this paper has been submitted to Astron. \& Astrophys. (E. Meyer-Hofmeister, N. Vogt and F. Meyer, "The influence of a magnetic field of the secondary star on dwarf nova outbursts", MPA Preprint no. 843, Dec. 1994). 